\title{
Gene ontology analysis of pairwise genetic associations in two genome-wide studies of sporadic ALS
}

\author{
Nora Chung Kim ${ }^{1}$, Peter C Andrews ${ }^{1}$, Folkert W Asselbergs ${ }^{3}$, H Robert Frost $^{1}$, Scott M Williams ${ }^{1}$, Brent T Harris ${ }^{4}$,
} Cynthia Read ${ }^{2}$, Kathleen D Askland ${ }^{2}$ and Jason H Moore ${ }^{1,2^{*}}$

\author{
* Correspondence: Jason.H.Moore@ \\ dartmouth.edu \\ ${ }^{1}$ Institute for Quantitative \\ Biomedical Sciences, Department of \\ Genetics, Dartmouth Medical \\ School, One Medical Center Dr., \\ Lebanon, NH 03756, USA \\ 2Department of Psychiatry and \\ Human Behavior, Butler Hospital, \\ Brown University, 345 Blackstone \\ Blvd, Providence, RI 02906, USA \\ Full list of author information is \\ available at the end of the article
}

\begin{abstract}
Background: It is increasingly clear that common human diseases have a complex genetic architecture characterized by both additive and nonadditive genetic effects. The goal of the present study was to determine whether patterns of both additive and nonadditive genetic associations aggregate in specific functional groups as defined by the Gene Ontology (GO).

Results: We first estimated all pairwise additive and nonadditive genetic effects using the multifactor dimensionality reduction (MDR) method that makes few assumptions about the underlying genetic model. Statistical significance was evaluated using permutation testing in two genome-wide association studies of ALS. The detection data consisted of 276 subjects with ALS and 271 healthy controls while the replication data consisted of 221 subjects with ALS and 211 healthy controls. Both studies included genotypes from approximately 550,000 singlenucleotide polymorphisms (SNPS). Each SNP was mapped to a gene if it was within $500 \mathrm{~kb}$ of the start or end. Each SNP was assigned a p-value based on its strongest joint effect with the other SNPs. We then used the Exploratory Visual Analysis (EVA) method and software to assign a p-value to each gene based on the overabundance of significant SNPs at the $a=0.05$ level in the gene. We also used EVA to assign $p$-values to each GO group based on the overabundance of significant genes at the $a=0.05$ level. A GO category was determined to replicate if that category was significant at the $a=0.05$ level in both studies. We found two GO categories that replicated in both studies. The first, 'Regulation of Cellular Component Organization and Biogenesis', a GO Biological Process, had p-values of 0.010 and 0.014 in the detection and replication studies, respectively. The second, 'Actin Cytoskeleton', a GO Cellular Component, had p-values of 0.040 and 0.046 in the detection and replication studies, respectively.
\end{abstract}

Conclusions: Pathway analysis of pairwise genetic associations in two GWAS of sporadic ALS revealed a set of genes involved in cellular component organization and actin cytoskeleton, more specifically, that were not reported by prior GWAS. However, prior biological studies have implicated actin cytoskeleton in ALS and other motor neuron diseases. This study supports the idea that pathway-level analysis of GWAS data may discover important associations not revealed using conventional one-SNP-at-a-time approaches.

\section{Biomed Central}

(c) 2012 Kim et al.; licensee BioMed Central Ltd. This is an Open Access article distributed under the terms of the Creative Commons Attribution License (http://creativecommons.org/licenses/by/2.0), which permits unrestricted use, distribution, and reproduction in any medium, provided the original work is properly cited. 


\section{Findings}

Amyotrophic Lateral Sclerosis (ALS) is a neurological disease that causes motor neuron degeneration, leading to paralysis and eventually death. Around 5,600 people are diagnosed with ALS each year with the incidence rate of two per 100,000 a year [1]. Despite the relatively low incidence rate compared to more prevalent diseases such as Alzheimer's and Parkinson's, ALS is a devastating disease with the average life expectancy of only two to five years from the time of diagnosis. Unfortunately, genome-wide association studies (GWAS) across multiple cohorts have not revealed replicable, genomewide significant single-nucleotide polymorphisms (SNP) associations that could provide additional clues about the etiology of ALS [2-7]. It is important to note that the prior studies assumed a simple genetic architecture with an analytical approach that was designed to only detect single SNPs with large effects independent of the genomic background or the ecological context of the subjects being studies. It is our working hypothesis that the genetic architecture of sporadic ALS is complex and likely to be influenced by gene-gene interactions [8]. We further hypothesize that patterns of gene-gene interactions influence ALS susceptibility at the pathway level with individual gene effects playing a smaller role. The goal of the present study was to determine whether patterns of pairwise genetic effects aggregate in specific functional groups as defined by the Gene Ontology (GO). We briefly outline here our bioinformatics approach and then summarize the findings.

Figure 1 provides a flowchart for our bioinformatics analysis. The analyses are split into three phases. The first phase is a SNP-level analysis that consists of data processing and gene-gene interaction analysis. The second phase is a gene-level analysis that determines whether each gene has more statistically significant SNPs than expected by chance. The third phase is a pathway-level analysis that determines whether each GO

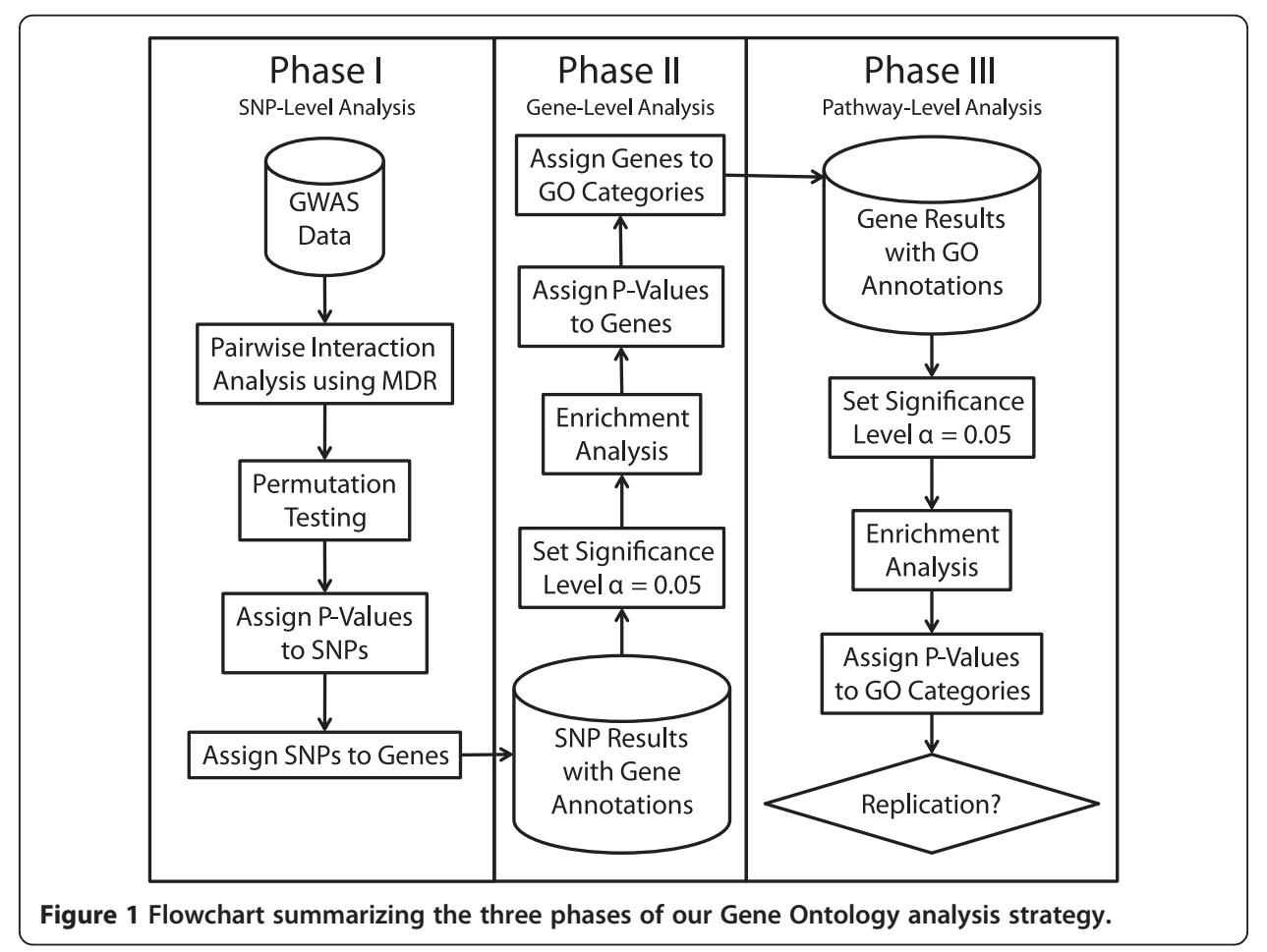


category has more statistically significant genes than expected by chance. This is followed by an assessment of replication between the independent GWAS studies of sporadic ALS. We briefly summarize the approach for each of these phases.

\section{Phase I: SNP-level analysis}

We ascertained and processed each GWAS dataset for sporadic ALS as described previously by Greene et al. [8]. The detection data consisted of 276 subjects with ALS and 271 healthy controls [3] while the replication data consisted of 221 subjects with ALS and 211 healthy controls [4]. Both studies included genotypes from approximately 550,000 single-nucleotide polymorphisms (SNPs). We then carried out a Multifactor Dimensionality Reduction (MDR) analysis to estimate the pairwise additive and nonadditive effects of SNPs on ALS susceptibility in each study. MDR is a machine learning method that was designed specifically to detect and characterize gene-gene interactions using constructive induction [9-12]. The MDR approach combines multiple SNPs at a time and then estimates the accuracy of a naïve Bayes classifier for predicting casecontrol status. The advantage of this approach is that it doesn't assume a particular genetic model and can thus simultaneously detect bot additive and nonadditive effects. Approximately 151 billion pairwise genetic effects were modeled in each data set.

Statistical significance of the accuracy of MDR models was evaluated using permutation testing. The goal of the permutation test was to estimate the distribution of MDR model accuracies under the null hypothesis of no association. Here, we randomized case-control labels 1000 times to create 1000 data sets consistent with the null hypothesis. In each null data set we estimated accuracies for all pairwise MDR models and selected the best one. Using this null distribution we derived the critical value of the accuracy statistic at an $\alpha=0.05$ significance level. Permutation testing revealed a critical value of the MDR classification accuracy of 0.629 for the detection data and 0.640 for the replication data at a genome-wide $\alpha=0.05$ significance level. This is a genome-wide significance level because it was derived from considering all possible pairs of SNPs with MDR on each null data set. Thus, the same numbers of models considered in the real data were also considered in each null data set. P-values were derived from the two null distributions for every MDR model in the detection and replication data sets.

The next step was to map the 151 billion pairwise MDR model p-values to each of the approximately 550,000 individual SNPs. We chose the best MDR model for each SNP and assigned that SNP the corresponding MDR model p-value. We then assigned SNPs to genes if they were within $500 \mathrm{~kb}$ upstream of a gene start or $500 \mathrm{~kb}$ downstream of a gene end. The rationale for this window size was to ensure inclusion of regulatory SNPs [13] that are expected to play an important role in gene-gene interactions due to effects of DNA sequence variation on transcriptional networks [14].

\section{Phase II: Gene-level analysis}

The gene-level enrichment analysis was performed using the Exploratory Visual Analysis (EVA) methodology and software. EVA was designed specifically for the visualization and analysis of statistical results from gene expression studies [15]. We have more recently expanded EVA for the analysis of SNP data $[16,17]$. The goal of phase II was to determine whether a gene has an overabundance of statistically 
significant SNPs. Here, we used EVA to assess whether SNPs with genome-wide significant MDR p-values of 0.05 or less are overrepresented given the size of the gene. Statistical significance is determined in EVA using a Fisher's exact test based on the hypergeometric distribution. P-values are then assigned to genes and genes assigned to GO categories. For the GO assignment, we used the annotations from the Molecular Signatures Database or MSigDB, version 3.0 [18].

It is important to note that a limitation of this approach is that it will likely miss pathways (GO categories) that are comprised mostly of genes that each has only one or a few significant SNPs. Our gene-level analysis makes the assumption that genes likely to show pathway-level effects (see Phase III below) are likely to be involved in multiple pairwise genetic associations within the same gene region and/or with other genes in the same pathway. Within a gene region, multiple SNPs in a promoter or enhancer sequence might synergistically influence the affinity of a protein to bind to DNA. It is also possible that one SNP influencing protein binding in a promoter might synergistically interact with another influencing protein binding at an enhancer through proteinprotein interactions and chromatin looping. Between two genes in different regions, synergy among SNPs could arise as the result of amino acid changes that change physical interactions of their corresponding protein products in a regulatory region of yet a third gene. Many more examples of regulatory sequence interactions are possible. We have previously speculated about such complex regulatory interactions [14].

\section{Phase III: Pathway-level analysis}

The pathway-level GO enrichment analysis was also performed using the EVA methodology and software as described above. Here, we used EVA to assess whether genes with p-values of 0.05 or less are overrepresented in a given pathway accounting for its size. A GO category was considered statistically significant at an $\alpha=0.05$ level. Gene Ontology categories that had p-values less than or equal to 0.05 in both the detection and replication datasets were considered replicated and carried forward for presentation and discussion. We explicitly used replication to control for false-positives due to multiple testing across GO categories.

\section{Results}

The bioinformatics analysis strategy described above and in Figure 1 revealed two replicated and statistically significant GO categories. The first, 'Regulation of Cellular Component Organization and Biogenesis', a GO Biological Process, had p-values of 0.010 and 0.014 in the detection and replication studies, respectively. The second, 'Actin Cytoskeleton', a GO Cellular Component, had p-values of 0.040 and 0.046 in the detection and replication studies, respectively. We focus here on interpretation of the role of actin cytoskeleton in sporadic ALS because it is a subcomponent of the set of cellular component organization and biogenesis genes.

\section{Discussion}

Actin is a highly conserved protein that forms microtubules in cells. Actin is an important part of the cytoskeleton and participates in or makes possible a number of cellular functions including protein trafficking and cell motility. The biological role of actin 
cytoskeleton in motor neuron diseases has long been recognized [19]. A recent review by Julien et al. [20] provides an overview of the many studies that have implicated cytoskeletal defects in ALS. Interestingly, SNPs in actin cytoskeleton genes have not replicated across the published GWAS for sporadic ALS [2-7]. They have, however, been implicated in other diseases such as multiple sclerosis [21]. Given the known biological basis for actin cytoskeleton in motor neuron diseases, including ALS, and the results presented here, we conclude that genes in this GO category should be examined more carefully in GWAS for sporadic ALS. In particular, such a careful examination should entail an analytical approach that is robust to the detection of pathway replication in the absence of gene replication. The results of this study support the idea that a bioinformatics approach to GWAS analysis that embraces the complexity of the genotypephenotype map has the potential to reveal interesting new associations that have not been discovered using one-SNP-at-a-time approaches.

\section{Availability}

The EVA and MDR software packages are freely available from the authors. More information can be found at http://www.epistasis.org.

Competing interests

The authors declare they have no competing interests.

\section{Authors' contributions}

NCK assisted with method development, carried out the analyses, interpreted the results and drafted the manuscript. PCA assisted with method development and with the programming necessary to implement the method. FWA, HRF, SMW, CR and KDA assisted with method development, interpreted the results and drafted the manuscript. BTH provided the data, interpreted the results and drafted the manuscript. JHM assisted with method development, assisted with the analyses, interpreted the results and drafted the manuscript.

\section{Acknowledgements}

This work was supported by NIH R01 grants LM010098, LM009012 and Al59694. NK was supported by a William H. Neukom Institute Fellowship at Dartmouth College and Howard Hughes Medical Institute Fellowship. FA was supported by a clinical fellowship from the Netherlands Organisation for Health Research and Development (ZonMw grant 90700342). The genotyping of samples was provided by the NINDS. The datasets used for the analyses described in this manuscript were obtained from the NINDS Database through dbGaP accession numbers phs000006.v1.p1 and pht000649.v1.p1. Permission to use these datasets was obtained through application to dbGAP by B.T.H.

\footnotetext{
Author details

${ }^{1}$ Institute for Quantitative Biomedical Sciences, Department of Genetics, Dartmouth Medical School, One Medical Center Dr., Lebanon, NH 03756, USA. ²Department of Psychiatry and Human Behavior, Butler Hospital, Brown University, 345 Blackstone Blvd, Providence, RI 02906, USA. ${ }^{3}$ Department of Cardiology, Division Heart \& Lungs, University Medical Center Utrecht, Utrecht, the Netherlands. ${ }^{4}$ Department of Neurology, 4000 Reservoir Rd, Georgetown University Medical Center, Washington, DC 20057, USA.
}

Received: 26 March 2012 Accepted: 13 July 2012 Published: 28 July 2012

References

1. ALS Association: http://www.alsa.org/about-als/facts-you-should-know.html.

2. Dunckley T, Huentelman MJ, Craig DW, Pearson JV, Szelinger S, Joshipura K, et al: Whole-genome analysis of sporadic amyotrophic lateral sclerosis. N Engl J Med 2007, 357(8):775-788.

3. Schymick JC, Scholz SW, Fung HC, Britton A, Arepalli S, Gibbs JR, et al: Genome-wide genotyping in amyotrophic lateral sclerosis and neurologically normal controls: first stage analysis and public release of data. Lancet Neurol 2007, 6(4):322-328.

4. Cronin S, Berger S, Ding J, Schymick JC, Washecka N, Hernandez DG, Greenway MJ, Bradley DG, Traynor BJ, Hardiman O: A genome-wide association study of sporadic ALS in a homogenous Irish population. Hum Mol Genet 2008, 17(5):768-774.

5. Cronin S, Tomik B, Bradley DG, Slowik A, Hardiman O: Screening for replication of genome-wide SNP associations in sporadic ALS. Eur J Hum Genet 2009, 17(2):213-218.

6. van Es MA, Veldink JH, Saris CG, Blauw HM, van Vught PW, Birve A, et al: Genome-wide association study identifies 19p13.3 (UNC13A) and 9p21.2 as susceptibility loci for sporadic amyotrophic lateral sclerosis. Nat Genet 2009, 41(10):1083-1087.

7. Laaksovirta H, Peuralinna T, Schymick JC, Scholz SW, Lai SL, Myllykangas L, et al: Chromosome 9p21 in amyotrophic lateral sclerosis in Finland: a genome-wide association study. Lancet Neurol 2010, 9(10):978-985. 
8. Greene $\mathrm{CS}$, et al: Multifactor dimensionality reduction for graphics processing units enables genome-wide testing of epistasis in sporadic ALS. Bioinformatics 2010, 26(5):694-695.

9. Ritchie MD, Hahn LW, Roodi N, Bailey LR, Dupont WD, Parl FF, Moore JH: Multifactor-dimensionality reduction reveals high-order interactions among estrogen-metabolism genes in sporadic breast cancer. Am J Hum Genet 2001, 69:138-147.

10. Ritchie MD, Hahn LW, Moore JH: Power of multifactor dimensionality reduction for detecting gene-gene interactions in the presence of genotyping error, missing data, phenocopy, and genetic heterogeneity. Genet Epidemiol 2003, 24:150-157.

11. Hahn LW, Ritchie MD, Moore JH: Multifactor dimensionality reduction software for detecting gene-gene and gene-environment interactions. Bioinformatics 2003, 19:376-382.

12. Moore JH, Gilbert JC, Tsai CT, Chiang FT, Holden T, Barney N, White BC: A flexible computational framework for detecting, characterizing, and interpreting statistical patterns of epistasis in genetic studies of human disease susceptibility. J Theor Biol 2006, 241:252-261.

13. Wang K, Li M, Bucan M: Pathway-based approaches for analysis of genomewide association studies. Am J Hum Genet 2007, 81:1278-1283.

14. Cowper-Sallari R, Cole MD, Karagas MR, Lupien M, Moore JH: Layers of epistasis: genome-wide regulatory networks and network approaches to genome-wide association studies. Wiley Interdiscip Rev Syst Biol Med 2011, 3:513-526.

15. Reif, et al: Exploratory visual analysis pharmacogenomic results. Pac Symp Biocomput 2005, :296-307.

16. Askland K, Read C, Moore JH: Pathways-based analyses of whole-genome association study data in bipolar disorder reveal genes mediating ion channel activity and synaptic neurotransmission. Hum Genet 2009, 125(1):63-79.

17. Askland K, Read C, O'Connell JH, Moore C: Ion channels and schizophrenia: a gene-set based analytic approach to GWAS data for biological hypothesis testing. Hum Genet 2012, 131(3):373-391.

18. Gillette MA, Paulovich A, Pomeroy SL, Golub TR, Lander ES, et al: Molecular signatures database (MSigDB) 3.0. Bioinformatics 2011, 27:1739-1740.

19. McMurray CT: Neurodegeneration: diseases of the cytoskeleton? Cell Death Differ 2000, 7(10):861-865.

20. Julien JP, Millecamps S, Kriz J: Cytoskeletal defects in amyotrophic lateral sclerosis (motor neuron disease). Novartis Found Symp 2005, 264:183-192.

21. Bush WS, McCauley JL, DeJager PL, Dudek SM, Hafler DA, Gibson RA, Matthews PM, Kappos L, Naegelin Y, Polman $\mathrm{CH}$, Hauser SL, Oksenberg J, Haines JL, Ritchie MD: International Multiple Sclerosis Genetics Consortium: A knowledge-driven interaction analysis reveals potential neurodegenerative mechanism of multiple sclerosis susceptibility. Genes Immun 2011, 12(5):335-340.

doi:10.1186/1756-0381-5-9

Cite this article as: Kim et al:: Gene ontology analysis of pairwise genetic associations in two genome-wide studies of sporadic ALS. BioData Mining 2012 5:9.

\section{Submit your next manuscript to BioMed Central and take full advantage of:}

- Convenient online submission

- Thorough peer review

- No space constraints or color figure charges

- Immediate publication on acceptance

- Inclusion in PubMed, CAS, Scopus and Google Scholar

- Research which is freely available for redistribution

Submit your manuscript at www.biomedcentral.com/submit 